\title{
Energy Efficient Anchor-Based Localization Algorithm for WSN
}

\author{
Tanuja Panda ${ }^{1}$, N.K. Kamila ${ }^{2}$, Rasmi Ranjan Patra ${ }^{3}$ \\ ${ }^{I}$ Dept. of CSE, C.V.Raman College of Engineering, INDIA \\ ${ }^{2}$ Dept. of CSE, C.V.Raman College of Engineering, INDIA \\ ${ }^{3}$ Dept. Of CSA, OUAT, INDIA
}

\begin{abstract}
Localization is one of the most fundamental problems in wireless sensor networks, since the locations of the sensor nodes are critical to both network operations and most application level tasks. To solve the localization problem for find out the location of sensor nodes in wireless sensor network, a Anchor based localization scheme for wireless sensor networks with energy efficiency is presented in this thesis. First, we develop a standardized clustering-based approach for the local coordinate system formation wherein a multiplication factor is introduced to regulate the number of cluster head nodes and neighbor nodes and the degree of connectivity among cluster head nodes. The algorithm operates asynchronously without a centralized controller; and in this algorithm location of the cluster heads be known a priori which are called anchor nodes. In this thesis to calculate the distance between the nodes triangulation technique is used. A simulation analysis on a specific numerical example is conducted for specifying the energy efficiency in finding the location by using anchor nodes. Experiments show that our algorithm beneficiary for less energy consumption.
\end{abstract}

\section{INTRODUCTION}

Wireless Sensor Network (WSN) is a special type of ad hoc network, where wireless devices get together and dynamically form a network without help of any infrastructure. It is composed of hundreds or thousands of low-cost sensing nodes, which are capable of performing limited sensing and communication tasks. Applications of this network vary from inhospitable habitats and disaster area to operating indoors for intrusion detection and equipment monitoring. The wireless micro sensors, which can be both a data source and a data router have been the topic of intensive research in recent years, are now emerging in several applications. Imagine a network of sensors sprinkled across a large building or an area such as a forest or battlefield. Typical tasks for such networks are to send a message to a node at a given location, even without knowing how many nodes there are or how to reach them, to retrieve sensor data (e.g., sound, light, radiation, temperature or humidity levels) from nodes in a given region, and to use the sensor nodes to track nearby events, such as vehicles moving through the sensor field. Most of these tasks require knowing the positions of the nodes or at least their relative positions. For example, for a vehicle-tracking application, the sensor nodes would determine the positions of the tracked vehicles relative to their own positions. With a network of thousands of nodes, it is unlikely that the position of each node can be precisely predetermined.

In many applications of wireless sensor networks, precise location information of sensor nodes is critical to the success of the applications. Most data collected from sensors are only meaningful when they are coupled with the location information of the corresponding sensors. Consider an application of habitat monitoring. Thousands of sensors are dropped in the targeted region of a tropical rain-forest by an aeroplane. Nodes are equipped with sensing devices to monitor the changes of temperature and humidity of the environment. To make every measurement useful to scientists, the location where measurements are taken has to be known.

Wireless sensor networks (WSNs) can work unattended for long periods, and find a very wide range of applications in the fields of environmental monitoring, forest fireproofing, biology habitat monitoring and control, intelligent agriculture, intelligent architecture and houses, defending military targets, preventing terror attacks, individual health monitoring, etc. [1].

The localization problem in wireless sensor networks is how to determine the location information of all or a subset of sensor nodes, given the measurements of pair wise spatial relationships between the nodes $[3,4]$. In the literature, WSNs localization methods can normally be categorized according to three different aspects: (i) the information requirements of the solution schemes: proximity-based localization, range-based localization, angle-based localization and probabilistic-based localization; (ii) the hardware requirements of the solution schemes: absolute localization and relative localization, and (iii) the type of network structure: static network and mobile network. 
In this paper, we present a GPS-based localization scheme for node localization in WSNs called the Energy efficient Anchor-based Localization Algorithm, where the task is to use the distance information between nodes to determine the coordinates of static nodes and also how much it is efficient for energy consumption is also determined. Since the sensors are battery operated so energy consumption is also most important while finding the location. When GPS is placed only for the cluster head nodes and other nodes can be find out their location by referencing that node is obviously consuming less energy. In this approach the triangulation technique is used to calculate the distance information obviously, our approach is a range-based relative localization algorithm applied in a stationary sensor network.

In this paper we make several departures from previous work. In the first phase, our contribution for developing a standardized process of constructing local coordinate systems is to exploit a new parameter, the multiplication factor $\alpha$, which can regulate the number of cluster head node and neighbor nodes in sensor networks and the degree of connectivity between cluster head nodes

Our approach can effectively overcome the time complexity problem taking into consideration using location information at one cluster head in the group of cluster nodes. A simulation analysis on a specific numerical example is conducted to overcome the energy consumption problem that happened in some previous works.

The remainder of the paper is organized as follows: In the following section we give an overview of the related work. In section 3, we provide a detailed description of our algorithm. Section 4 describe the theoretical analysis about the energy consumption. The section 5 shows some simulation results specifying energy consumption and the next final section concludes the paper.

\section{RELATED WORK}

The localization problem consists in finding the geographic location of nodes in a WSN. The location of a node can be computed by a central unit (the sink node) [8] or in a distributed manner [9, 10, 11], which is frequently used. Essentially, the location discovery problem can be split into two stages: distance estimation and position computation [12]. Usually, the distance between two nodes is estimated based on methods such as Received Signal Strength Indicator (RSSI), Time of Arrival (ToA), and Time Difference of Arrival (TDoA) [13]. Once the distance is estimated, at least three methods can be used to compute the node location: Triangulation, Trilateration, and Multilateration [14]. Triangulation is used when we have the directional information instead of the distance information, which is the case of Angle of Arrival (AoA) systems in which the angle of arrival of the received signal is estimated and the node position is computed through trigonometrical relations (Fig 1(a)). Trilateration is the most basic and intuitive method that locates a node by computing the intersection of three circles (Fig 1(b)); in this case, the location is found by solving a simple linear system. In Multilateration, the node position is estimated by minimizing the differences between the measured distances and estimated distances (Fig 1(c)); in this case, the location is found by solving an overdetermined linear system. A node that have at least three reference neighbors can estimate its own location through trilateration. Then, this node becomes a reference by broadcasting its location and helping other nodes to estimate their own locations.

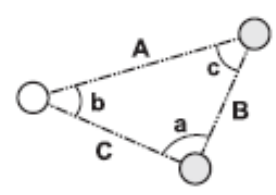

(a)

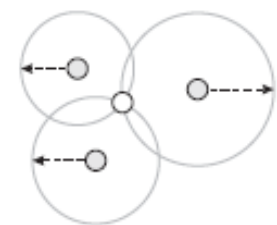

(b)

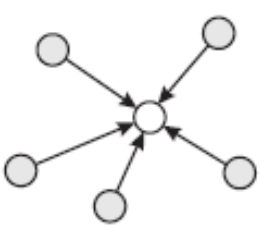

(c)

Figure 1: "Position estimation methods: (a) triangulation, (b) trilateration, and (c) multilateration"

Node localization has been the topic of active research and a number of systems have been proposed over the past few years. Many of those systems fall into one of three classes or a combination of them. The first class includes range free algorithms, which assume that there is no distance/angle information available at each node $[15,16]$.

In general, range-free techniques provide the lowest level of accuracy among the three classes. The second class employs a number of specialized, anchor, nodes that know their positions usually using GPS $[17,18,19]$. The rest of the nodes try to estimate their positions relative to these anchors. For example, in the iterative multilateration method, an unknown node that is connected to at least three anchors estimates its position by solving a system of equations. Once a node estimates its position it becomes an anchor and assists other unknown nodes in estimating their positions by propagating its own location estimate through the network. 
Most of the methods in this class require a high percentage of anchor nodes. The third class of localization systems tries to compute nodes' positions without the use of anchor nodes. In this case, instead of computing absolute node positions, the algorithm estimates nodes' positions relative to a coordinate system established by a reference group of nodes. Relative positioning can be sufficient for many applications to work efficiently; Schemes in this class can be range-free or range-based. Algorithms are also developed for node localization which uses anchor-free concept and using the triangulation method for easy estimation of node position [22].

In previous research so many techniques are used to find about the location information. In this thesis we used the range based \& anchor based localization algorithm for sensor networks. Here we build a cluster-wide coordinate system, estimates the position of one-hop neighbors using triangulation and broadcasts this information to all the nodes in the network to build a global network coordinate system. Instead of forming a local coordinate system at each node, we build a cluster-wide coordinate system at each cluster head. In this case we gain the following benefits: (1) since the cluster head node is known its position so its easy to find out the other position using triangulation technique with less time consumption.; (2) the communication overhead to build global network topology is reduced since only cluster head communicate with each other.

We estimate the position of nodes that are within k-hops from the cluster head node since the cluster head serves the entire cluster and also finding the energy consumption by some simulation work.

\section{ENERGY EFFICIENT ANCHOR-BASED LOCALIZATION ALGORITHM}

\subsection{Assumptions and Scheme Overview}

In this thesis, we investigate a GPS-based node localization algorithm for WSNs. It is assumed that distance measurements are already made hop-to-hop between neighboring sensor nodes. There are a number of technologies and techniques for performing the distance measurements. The simplest way is to measure the received signal strength and then apply a path loss model, such as the log path loss model [21] to calculate the distance. The distance can also be determined based on the time-of-arrival measurements and the measurements of round-trip time-of-flight of a radio signal. When both a radio signal and an ultrasound signal are employed, an extremely accurate time-of-flight estimate and hence distance estimate can be obtained. We make the following main assumptions on our model:

- All sensors are stationary. So the network topology is fixed.

- There is one landmark in network. That is to say, the cluster head sensor has the location information which are called anchor nodes.

- All sensors are homogeneous, with the same technical characteristics, and especially the same transmission range.

- $\quad$ All sensors have enough energy to accomplish a node localization algorithm.

- $\quad$ All sensors use omnidirectional antennae.

- $\quad$ All the wireless links between sensors are bidirectional.

- There are no base stations to coordinate or supervise activities among sensors. Hence, the sensors must make all decisions without reference to a centralized controller.

In a stationary wireless sensor network, as sensors are battery-constraint devices, network topology may change in two cases: some sensors will die away or go to sleep periodically, and some sensors will be resupplied to maintain the work efficiency and quality of the whole network. In general, we run our proposed algorithm when all sensor nodes are deployed in some area. After that, the location information of all sensor nodes will remain during all lifetime of sensor networks even some sensor nodes die due to energy exhaustion or go to sleep periodically to save their energy. In the latter case, we can use other absolute localization approaches to determine the coordinates of new sensor nodes because the former sensor nodes which know their global coordinate now can be considered as beacons. The simplest and the most widely used absolute localization approach is trilateration, which is suitable for our purposes because the number of new sensor nodes is trivial compared with hundreds and even thousands of former sensor nodes.

\subsection{Construction of Local Coordinate Systems}

Scalability and the need to conserve energy and reduce communication overhead led to the idea of organizing the sensors hierarchically, which can be accomplished by gathering collections of sensors in to clusters [23]. Clustering sensors, which are the core ideas of this algorithm and its improved algorithms, are advantageous because they: (i) conserve limited energy resources and improve energy efficiency, (ii) aggregate information from individual sensors and abstract the characteristics of network topology, (iii) provide scalability and robustness for the network.

In the following discussion, we assume that a number of sensors are deployed randomly over a geographical region with a given average density. After deployment, each sensor starts to decrement a random waiting timer. 
If the timer of node ' $i$ ' expires, then the sensor ' $\mathrm{i}$ ' broadcasts a message $\mathrm{M}_{1}$ with a multiplication factor $\alpha \geq 1$ proclaiming that it is beginning a Cluster Head, a focal point of a new cluster. All nodes in the communication range of node ' $i$ ' who receives this message become a neighbor node. We refer to some nodes, which hear from other cluster head nodes as border nodes. At the same time, all nodes hearing the message M1 also transmit messages M2 to their neighbor nodes announcing their existence and the distance between them. However, events may occur and cause a sensor to extend or stop its timer. For example, if a neighbor declares itself to be a cluster head node, the sensor lengthens the timer. On the other hand, whenever the timer is greater than threshold value, the sensor cancels its own timer. The complete procedure of the clustering phase is outlined in the proposed Algorithm. After clustering phase, there are three different kinds of sensor: cluster head nodes, neighbor nodes and border nodes. (Fig 2)

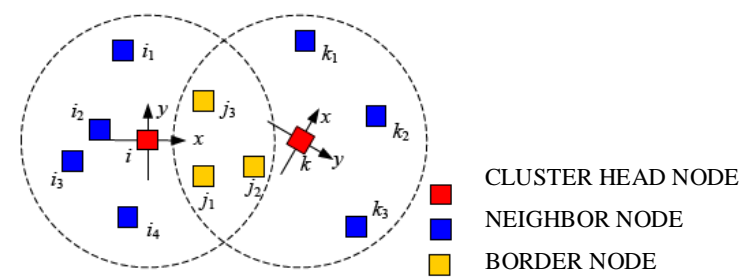

Figure 2: "Finding different nodes after establishing local coordinate system"

\subsection{Algorithm}

Step -1: One node have the position information \& that named as $\mathrm{CH}$.

Step - 2: Each other node initializes a random waiting time

$\mathrm{Wt}_{\mathrm{i}}^{(0)} \in\left(0, \mathrm{~T}_{\max }\right)$

Step - 3: Decrease all random waiting timer $\mathrm{Wt}_{0}{ }^{(\mathrm{k})}$.

Step - 4: CH node broadcast a message M1 with a multiplication factor $\alpha$.

Step - 5: if a sensor $\mathrm{S} 1$ receive the message M1 at time step k

(a) $S_{j}=$ Neighbor Node

(b) transmit message M2 to its cluster head \& neighbor nodes with the distance between i \& j.

(c ) $\mathrm{Wt}_{\mathrm{j}}^{(\mathrm{k}+1)}=\alpha * \mathrm{Wt}_{\mathrm{j}}^{(\mathrm{k})}$

(d) If $\mathrm{Wt}_{\mathrm{ij}}^{\left({ }^{(\mathrm{k}+1)}\right.}>\mathrm{T}_{\max }$ delete the waiting time. end

end

Step - 6: If the waiting time of all sensor nodes are deleted

algorithm is over.

else

$\mathrm{k}=\mathrm{k}+1 \&$ go to step (2)

end

Assume that the multiplication factor $\alpha=\infty$, once a sensor receives a message M1 transmitted from cluster head node $\mathrm{CH}$ at time step $k$, the random waiting timers of all neighbor nodes will be greater than the threshold value Tmax, and the neighboring sensors will not be Cluster Head nodes any more. As a result, all Cluster Head nodes are not connected in clustering sensor networks as in the literature. In another special case of Algorithm when the multiplication factor $\alpha=1$, the waiting timers of all nodes evidently will not change during one step under the same conditions. As time goes on, all nodes will inevitably become Cluster Head node. There are, in essence, no concepts of Neighbor nodes and border nodes in Algorithm like in the literature. Let us consider another case in which the multiplication factor $\alpha$ is related to the distance between two nodes $i$ and $j$, that is $\alpha=f(d i j)$, and the more dij is, the less $\alpha$ is. In this case, each Cluster Head node has a greater chance of being deployed in the range of another Cluster Head node.

It is worth noting that more neighbor nodes can easily find out during the early periods in Algorithm, while during the late ones, on the contrary, it is hard for neighbor nodes to become Neighbor nodes. In order to avoid oscillation and keep the number of Neighbor nodes and cluster head nodes stable, one of the most viable solutions is the use of time factor in $\alpha$ to provide a little $\alpha(t)$ during the early stages and a large $\alpha(t)$ during the later stages.

The above implies that in the clustering phase, the multiplication factor $\alpha$ plays an important role in adjusting the number of cluster head nodes and Neighbor nodes and the degree of connectivity among Cluster Head nodes 
and reducing communication overhead. It is reasonable to investigate the performance from the perspective of multiplication factor. In this thesis, we use triangulation to form the local coordinate system at each Cluster Head node like in other relative localization algorithms. Fig 2 depicts typical runs after the local coordinate system is established. The results show that all nodes in the sensor network are divided into three different kinds of sensor: Cluster Head nodes, Neighbor nodes and border nodes, the relationships between which have a significant impact on the localization.

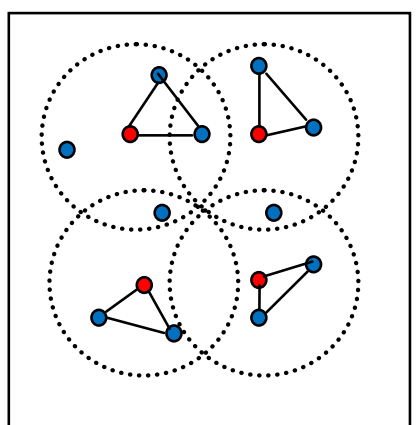

Cluster Head nodes

Neighbor nodes

Figure 3 : "Different cluster of nodes creating triangles for finding position"

\subsection{Mathematical Formulation (for Distance Calculation)}

In this section we show how each Cluster Head node builds its local coordinate system. The cluster head becomes the center of its own coordinate system with the position $(0,0)$ and the positions of its neighbor nodes are computed accordingly.

The node $\boldsymbol{b}$ is called a one-hop neighbor of node $\boldsymbol{a}$ if nodes $\boldsymbol{a}$ and $\boldsymbol{b}$ can communicate directly ( in one hop). Let $\mathbf{N}$ be the set of all the nodes in the network. We define for all $\boldsymbol{a} \varepsilon \mathbf{N}$, a set of nodes $\mathbf{K}_{\mathbf{a}}$ such that for all $\boldsymbol{b} \varepsilon \mathbf{K}_{\mathrm{a}}, \mathrm{b} \neq \boldsymbol{a}, \boldsymbol{b}$ is a one hop neighbor of $\boldsymbol{a}$. We call $\mathbf{K}_{\mathrm{a}}$ the set of one-hop neighbors of node $\boldsymbol{a}$.

The distance between any two nodes, $\boldsymbol{c} \& \boldsymbol{d}$ are denoted by $\boldsymbol{d}_{\boldsymbol{c} \boldsymbol{d}}$. A coordinate system can then be established, if there exists two nodes $\boldsymbol{c}, \boldsymbol{d} \varepsilon \mathbf{K}_{\mathrm{a}}$ such that $\boldsymbol{d}_{\boldsymbol{c} \boldsymbol{d}}$ is known at node $\boldsymbol{a}$. With the cluster head $\boldsymbol{a}$ being the origin of the coordinate system, either node $\boldsymbol{c}$ (or node $\boldsymbol{d}$ ) can be defined to lie on the positive $\mathrm{x}-$ axis.

Node $\boldsymbol{d}$ (or node $\boldsymbol{c}$ ) is now assumed to have a positive $\boldsymbol{y}$ component to define the $\mathrm{Y}-$ axis and the coordinates of the nodes, $\boldsymbol{a}, \boldsymbol{c}$ and $\boldsymbol{d}$ are given by :

$\mathrm{a}_{\mathrm{x}}=0, \mathrm{a}_{\mathrm{y}}=0$;

$\mathrm{c}_{\mathrm{x}}=\mathrm{d}_{\mathrm{ac}}, \mathrm{c}_{\mathrm{y}}=0$;

$\mathrm{d}_{\mathrm{x}}=\mathrm{d}_{\mathrm{ad}} \cos \gamma, \mathrm{d}_{\mathrm{y}}=\mathrm{d}_{\mathrm{ad}}^{2} \sin \gamma$

Where $\gamma$ is the angle $<(\mathrm{c}, \mathrm{a}, \mathrm{d})$ in the triangle $\Delta(\mathrm{c}, \mathrm{a}, \mathrm{d}) \&$ it is obtained by using a cosine rule for triangle. $\gamma=\arccos \left(\mathrm{d}_{\mathrm{ad}}{ }^{2}+\mathrm{d}_{\mathrm{ac}}{ }^{2}+\mathrm{d}_{\mathrm{cd}}{ }^{2}\right) / 2 \mathrm{~d}_{\mathrm{ad}} \mathrm{d}_{\mathrm{ac}}$

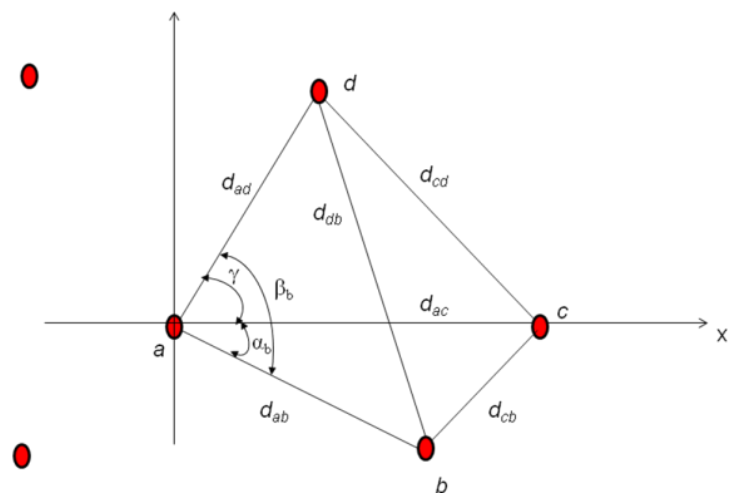

Figure 4: "An example illustrating the way to obtain the position of node $j$ in the coordinate system of node $i$."

The position of other nodes $\mathbf{b}, \mathbf{b} \varepsilon \mathbf{K}_{\mathrm{a}}, \mathrm{b} \neq \mathrm{c}, \mathrm{d}$ for which $d_{a b}, d_{d b}, d_{c b}$ are known, are computed by triangulation. Therefore, we obtain:

$\mathrm{b}_{\mathrm{x}}=\mathrm{d}_{\mathrm{ab}} \cos \alpha_{\mathrm{b}}$ 
$b_{y}= \begin{cases}d_{a b} \sin \alpha_{b} & \text { if } \beta_{b}=\alpha_{b}-\gamma \\ -d_{a b} \sin \alpha_{b} & \text { else }\end{cases}$

When $\alpha_{\mathrm{b}}$ is the angle $<(\mathrm{c}, \mathrm{a}, \mathrm{b})$ in the triangle $\Delta(\mathrm{c}, \mathrm{a}, \mathrm{b}) \& \beta_{\mathrm{b}}$ is the angle $<(\mathrm{b}, \mathrm{a}, \mathrm{d})$ in the triangle $\Delta(\mathrm{b}, \mathrm{a}, \mathrm{d})$. Then we obtain the values of $\alpha_{b} \& \beta_{b}$ by using the cosine rule :

$\alpha_{\mathrm{b}}=\arccos \left(\mathrm{d}_{\mathrm{ab}}{ }^{2}+\mathrm{d}_{\mathrm{ac}}{ }^{2}+\mathrm{d}_{\mathrm{bc}}{ }^{2}\right) / 2 \mathrm{~d}_{\mathrm{ab}} \mathrm{d}_{\mathrm{ac}}$

$\beta_{b}=\arccos \left(d_{a b}+d_{a d}^{2}+d_{b d}^{2}\right) / 2 d_{a b} d_{a d}$

The positions of the nodes $\mathrm{k} \varepsilon \mathrm{K}_{\mathrm{a}}, \mathrm{k} \neq \mathrm{c}$, d, and $\mathrm{k} \varepsilon \mathrm{K}_{\mathrm{c}}, \mathrm{k} \varepsilon \mathrm{K}_{\mathrm{d}}$, can be computed by using the positions of the node $\mathrm{a}$ and at least two other nodes for which the positions are already obtained, if the distance from the node $\mathrm{k}$ to these nodes is known.

\subsection{Analysis of energy Consumption}

\section{THEORITICAL ANALYSIS}

The total power requirements include both the power required to transmit messages and the power required to receive (or process) messages. When a sensor, say sensor ' $\mathrm{i}$ ', considered as cluster head node, it broadcast message M1 \& assigns cluster ID i to its neighboring sensors. Its neighboring sensors then transmit a signal M2 to their neighbor nodes with the distance information.

During this clustering phase, $\left(1+\mathrm{N}_{\mathrm{i}}\right)$ transmission and $\left(\sum_{j \in \mathrm{Ci}} \mathrm{Nj}+\mathrm{Ni}\right)$ receptions are executed where $\mathrm{N}_{\mathrm{i}}$ is the no. of neighbor nodes of sensor $i$, and $\mathrm{C}_{\mathrm{i}}$ is the index set of neighboring sensors of sensor $\mathrm{i}$. This procedure is applied to all cluster head nodes and their neighbor nodes.

Now let $\mathrm{N}_{T}$ and $\mathrm{N}_{\mathrm{R}}$ denote the total number of transmissions and reception for all cluster head nodes respectively. Hence:

$$
\begin{aligned}
& \mathrm{N}_{\mathrm{T}}=\sum_{i \in I}(1+\mathrm{Ni}) \\
& \mathrm{N}_{\mathrm{R}}=\sum_{i \in I}\left(\sum_{j \in \mathrm{Ci}} \mathrm{Nj}+\mathrm{Ni}\right)
\end{aligned}
$$

Where $\mathrm{I}$ is the index set of cluster head nodes in the WSNs. Suppose $\mathrm{E}_{\mathrm{T}}$ is the energy needed to transmit, which depends on the node communication redius $R$ and $E_{R}$ is the energy needed to receive. So from (1) and (2), the total energy consumption, $\mathrm{E}_{\text {total }}$ for cluster of WSN is

$$
E_{\text {total }}=N_{T} \cdot E_{T}+N_{R} \cdot E_{R}
$$

The above analysis is suitable for any transmitting range. However, overly small transmission ranges may result in isolated clusters whereas overly large transmission ranges may result in a single cluster.

Therefore, in order to optimize energy consumption and encourage linking between clusters, it is sensible to consider the minimum transmission power which will result in a fully connected network.

\section{EXPERIMENTAL RESULTS}

\subsection{Performance Evaluation}

We have validated our anchor-based localization algorithm using simulation. We validate it by considering the energy consumption as main factor. There is one parameters used in our simulation: i.e. $\alpha$. We also consider the accuracy of the estimated positions as a performance metric.

The algorithm was implemented using MATALB 7.0. All experiments were performed over 1000 homogeneous nodes making different clusters of various ranges. The cluster radius ranges from $2 \mathrm{~m}$ to $5 \mathrm{~m}$ depending on the cluster size and node connectivity. The nodes were randomly placed according to a uniform distribution on a $100 \times 100$ area.

As discussed in section 4.4, the local coordinate system is determined by assuming the cluster head node ' $\boldsymbol{a}$ ' at the origin and selecting two reference nodes ' $\boldsymbol{b}$ ', ' $\boldsymbol{c}$ ' that are adjacent to each other and to ' $\boldsymbol{a}$ ' and the three nodes are not collinear with each other. The three nodes together form a triangle as shown in Fig. 4 in the section 3.4 .

\subsection{Simulation Results}

In the result two graph figures are developed by putting equation in anchor based localization algorithm. The Fig-5 shows the energy consumption in joule for different nodes range from 100 to 1000 making different clusters of node. The Fig- 6 shows the energy consumption at different cluster of nodes before 
applying the algorithm and after applying the algorithm. The result shows that after applying the algorithm the energy consumption value is less than that of before.

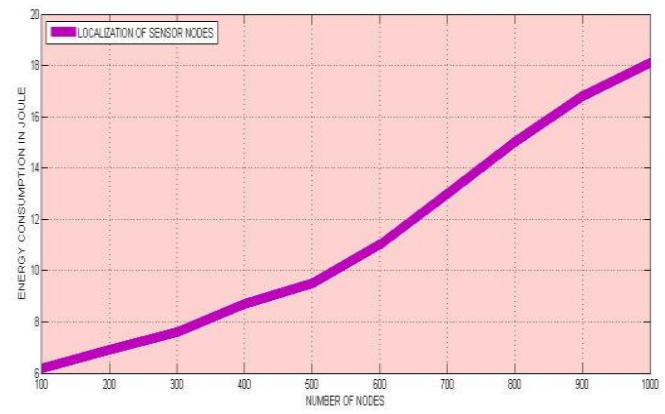

Figure 5 : "Energy consumption in Joule at different nodes"

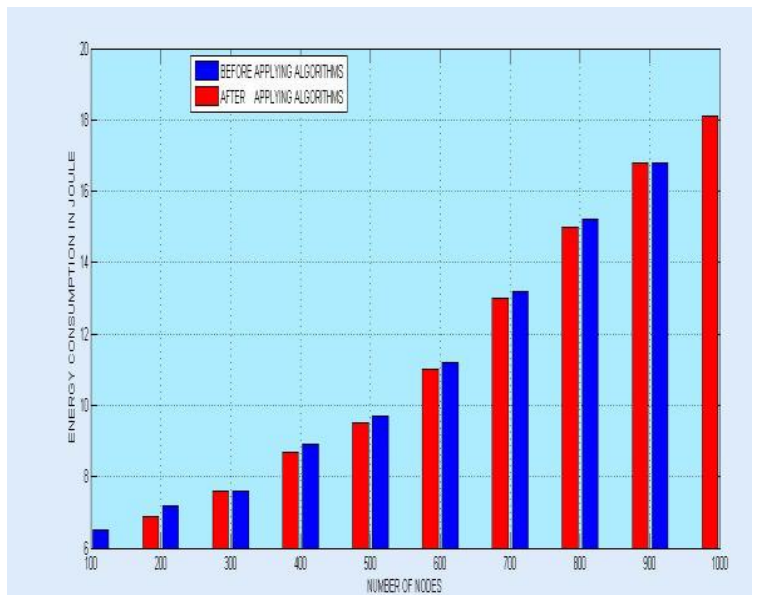

Figure 6: "Energy consumption in Joule at different nodes before applying and after applying the algorithms"

\section{CONCLUSION \& FUTURE WORK}

This paper has presented a GPS-based localization scheme with energy efficiency for wireless sensor networks, which has fixed anchor nodes. The proposed anchor based localization algorithm combines a standardized clustering based approach to construct local coordinate systems. The effectiveness of the proposed algorithm has been demonstrated through simulation and investigated the energy requirements.

Despite the results obtained in this paper give positive answers to a number of the problems that motivated our study, there are still many unsolved problem in the area. Since the sensors are equipped with limited energy supplies, one of the primary goals is to reduce the overall energy consumption of the network, thus increasing its lifetime. As part of our continuing research, we plan to discuss more technical issues about the energy consumption problem to verify the performance of the proposed scheme. Additionally, we are interested in testing and improving the performance of our algorithm under distance measurement errors so that it will be more feasible in real world applications. Finally, idea can be extended for three dimensional model and mobile wireless sensor networks.

\section{REFERENCES}

[1] Dachrach, J.; Taylor, C. Localization in sensor networks. In Handbook of Sensor Networks: Algorithms and Architectures; (Stojmenovic, I., Ed.; John Wiley and Sons: Hoboken, NJ, USA, 2005.)

[2] Tilak, S., Abu-Ghazaleh, N. B., and Heinzelman, W. A taxonomy of wireless micro-sensor network models. (Mobile Computing and Communications Review 2002)., 9 28-36,

[3] Mao, G.Q.; Fidan, B.; Anderson B.D.O. Wireless sensor network localization techniques (Comput. Networks, 2007) 51, 25292553 ,.

[4] Wang, J.; Ghosh, R.K.; Das, S.K. A survey on sensor localization. J. Control Theory Appl 8 2010, 2-11.

[5] Shah, R. C., and Rabaey, J. M. Energy aware routing for low energy ad-hoc sensor networks. In Proceedings of the 3rd IEEE Wireless communications and Networking Conference, Orlando, FL, 2001, 151-165. 
[6] Megeurdichian, S., Slijepcevic, S., Karayan, V., and Potkonjak, M. Localized algorithms in wireless ad-hoc networks:Location discovery and sensor exposure, MobiHOC, 106-116, 2001.

[7] Bordim, J. L., Nakano, K., and Shen, H. Sorting on a single channel wireless sensor networks. In Proceedings of the International Symposium on Parallel Architectures and Networks, 2002, 153-158.

[8] L. Doherty, K. S. Pister, and L. E. Ghaoui, Convex position estimation in wireless sensor networks in INFOCOM 2001, vol. 3, Anchorage,AK, USA, , April 2001 pp. 1655-1663.

[9] A. Savvides, C.-C. Han, and M. B. Strivastava, Dynamic fine-grained localization in ad-hoc networks of sensors in 7th ACM/IEEE International Conference on Mobile Computing and Networking, Rome, Italy, 2001 pp. 166-179.

[10] D. Niculescu and B. Nath, Ad hoc positioning system (aps) in IEEE Global Communications Conference (GlobeCom '01), no. 5, San Antonio, TX, USA, , November 2001pp. 2926-2931.

[11] N. Bulusu, J. Heidemann, D. Estrin, and T. Tran, Self-configuring localization systems: Design and experimental evaluation Trans. on Embedded Computing Systems, vol. 3, no. 1, February 2004 pp. 24-60.

[12] C. Savarese, J. M. Rabaey, and J. Beutel, Locationing in distributed adhoc wireless sensor networks in Proc. of the IEEE Signal Processing Society International Conference on Acoustics, Speech, and Signal Processing 2001 (ICASSP 2001), vol. 4, Salt Lake City, UT, USA, May 2001 pp. 2037-2040.

[13] J. D. Gibson, Ed., The Mobile Communication Handbook IEEE Press, 1999.

[14] A. Savvides, C.-C. Han, and M. B. Strivastava, Dynamic fine-grained localization in ad-hoc networks of sensors in 7th ACM/IEEE International Conference on Mobile Computing and Networking, Rome, Italy, 2001, pp. 166-179.

[15] L. Doherty, K. Pister, and L. El Ghaoui, Convex Position Estimation in Wireless Sensor Networks in the Proceedings of lEEE Conference oii Cornpier Co nniunica ions (iNFOCOM), Anchorage, Alaska, April 2001,

[16] N. Bulusu, J. Heidemann, and D. Estrin, GPS-less Low-cost Outdoor Localization for Very Small Devices ZEEE Personal Corizmruiicatiuns, October 2000, 7(5):2\&34

[17] X. Cheng, A. Thaeler, G. Xue, and D. Chen, TPS: A Time- Based Positioning Scheme for Outdoor Sensor Networks in the Proceedirrgs of IEEE Conference 011 COlnpuler Comtnunications (INFOCOM), Hong Kong, March 2004.

[18] A. Savvides, H. Park, and M. B. Srivastava, The Bits and Flops of The n-hop Multilateration Primitive for Node Localization Problems in the Proceedings of [lie first ACM international niorkshop on Wireless Setisor Netwoi-ks and Applications, Atlanta, GA, September 2002.

[19] A. Sawides, C. C. Han, and M. Srivastava, Dynamic Fine-Grained Localization in Ad-hoc Networks of Sensors in the Proceedirigs of the rh IEEE/ACM Annual Conference onMobile Coniputirrg and Networks (hfobiCUM'Ol), Rome,Italy, July 2001.

[20] Rappaport, T.S. Wireless Communications: Principles and Practice, 2nd ed.; (Prentice Hall: Englewood Cliffs, NJ, USA, 2001).

[21] Wen, C.Y.; Hsiao, Y.C. Decentralized anchor-free localization for wireless ad-hoc sensor networks. In Proceedings of 2008 IEEE International Conference on System, Man, and Cybernetic. Singapore, October 2008 pp. 2776-2784.

[22] Adel Youssef, Ashok Agrawala, Mohamed Younis Accurate Anchor-Free Node Localization in Wireless Sensor Networks in the Proceedings of the IEEE International Conference on System, 2005, pp- 465-470.

[23] Wen, C.Y.; Sethares, W.A. Automatic decentralized clustering for wireless sensor networks. Eurasip J. Wirel. Comm. 2005, 28, 686-697.

[24] I.F. Akyildiz, W. Su*, Y. Sankarasubramaniam, E. Cayirci Wireless sensor networks: a survey I.F. Akyildiz et al. / Computer Networks (Published by Elsevier Science B.V.)38 (2002) 393-422

[25] Asis Nasipuri and Kai Li A Directionality based Location Discovery Scheme for Wireless Sensor Networks WSNA'02 September 28, 2002, Atlanta, Georgia, USA.

[26] 26. Jonathan Bachrach and Christopher Taylor Localization in Sensor Networks pp-i-xxxviii.

[27] Thomas J Stevens, Darryl Morrell Minimization of sensor usage for target tracking in a network of irregularly spaced sensors.

[28] H. Yang and B. Sikdar A Protocol for Tracking Mobile Targets using Sensor Networks

[29] Yoshikazu Ohta, Masashi Sugano, Masayuki Murata, Autonomous Localization Method inWireless Sensor Networks Proceedings of the 3rd Int'l Conf. on Pervasive Computing and Communications Workshops (PerCom 2005 Workshops) 0-7695-2300-5/05 $\$ 20.00$ (C) 2005 IEEE.

[30] Neal Patwari, Joshua N. Ash, Spyros Kyperountas, Alfred O. Hero III, Randolph L. Moses, and Neiyer S. Correal Locating the Nodes Cooperative localization in wireless sensor networks IEEE SIGNAL PROCESSING MAGAZINE, JULY 2005 PP - 54-69.

[31] Jie Liu, Patrick Cheung, Leonidas Guibas, Feng Zhao A Dual-Space Approach to Tracking and Sensor Management in Wireless Sensor Networks Palo Alto Research Center Technical Report, March 2002 P2002-10077, p 1-9, 